\title{
Polarization Observations with the Cosmic Background Imager
}

\author{
A. C. S. Readhead, ${ }^{1 *}$ \\ S. T. Myers, ${ }^{2}$ \\ T. J. Pearson, ${ }^{1}$ \\ J. L. Sievers, ${ }^{1,3}$
B. S. Mason, ${ }^{4} \quad$ C. R. Contaldi, ${ }^{3} \quad$ J. R. Bond ${ }^{3} \quad$ R. Bustos,${ }^{5} \quad$ P. Altamirano, ${ }^{6}$
C. Achermann, ${ }^{6}$
L. Bronfman, ${ }^{6}$
J. E. Carlstrom, ${ }^{7}$
J. K. Cartwright, ${ }^{1,7}$
S. Casassus, ${ }^{6}$
C. Dickinson, ${ }^{1}$
W. L. Holzapfel, 8
J. M. Kovac, ${ }^{1,7}$ \\ E. M. Leitch, ${ }^{7} \quad$ J. May, ${ }^{6}$ \\ S. Padin, ${ }^{1,7}$ \\ D. Pogosyan, ${ }^{3,9}$ \\ M. Pospieszalski, ${ }^{10}$
C. Pryke, ${ }^{7}$
R. Reeves, ${ }^{5}$
M. C. Shepherd, ${ }^{1}$ \\ S. Torres ${ }^{5}$ \\ ${ }^{1}$ Owens Valley Radio Observatory, California Institute of Technology, Pasadena, CA 91125, USA. \\ ${ }^{2}$ National Radio Astronomy Observatory (NRAO), Post Office Box O, Socorro, NM 87801, USA. \\ ${ }^{3}$ Canadian Institute for Theoretical Astrophysics (CITA), University of Toronto, \\ Toronto, Ontario, M5S3H8, Canada. \\ ${ }^{4}$ NRAO, Post Office Box 2, Green Bank, WV 24944, USA \\ ${ }^{5}$ Departamento de Ingeniería Eléctrica, Universidad de Concepción, Concepción, Chile. \\ ${ }^{6}$ Departamento de Astronomía, Universidad de Chile, Santiago, Chile. \\ ${ }^{7}$ Kavli Institute for Cosmological Physics, Department of Astronomy and Astrophysics, \\ University of Chicago, Chicago, IL 60637, USA. \\ ${ }^{8}$ Department of Physics, 361 LeConte Hall, University of California, Berkeley, CA 94720-7300. \\ ${ }^{9}$ Department of Physics, University of Alberta, Edmonton, Alberta, T6G2J1, Canada. \\ ${ }^{10}$ NRAO, 520 Edgemont Road, Charlottesville, VA 22903, USA. \\ *To whom correspondence should be addressed; E-mail: acr@astro.caltech.edu.
}

Science 306: 836-844. Published online October 7, 2004; 10.1126/science.1105598

Polarization observations of the cosmic microwave background with the Cosmic Background Imager from September 2002 to May 2004 provide a significant detection of the E-mode polarization and reveal an angular power spectrum of polarized emission showing peaks and valleys that are shifted in phase by half a cycle relative to those of the total intensity spectrum. This key agreement between the phase of the observed polarization spectrum and that predicted on the basis of the total intensity spectrum provides support for the standard model of cosmology, in which dark matter and dark energy are the dominant constituents, the geometry is close to flat, and primordial density fluctuations are predominantly adiabatic with a matter power spectrum commensurate with inflationary cosmological models.

In recent years a wide variety of observations have provided support for a standard model of cosmology and cosmic structure formation. In this model (1] 2 2 3), the mass-energy density of the universe is dominated by cold dark matter and dark energy, possibly in the form of Einstein's cosmological constant, and 
conventional baryonic matter is only a minor component. As the universe expanded from its hot, dense origins, all the structures seen in the universe today formed under the action of gravity on initial nearly scale-invariant adiabatic gaussian density fluctuations. Observations of the anisotropy in the intensity of the cosmic microwave background radiation (CMB) have provided much of the evidence for this model and estimates of the values of the fundamental parameters, including the spatial curvature and the densities of dark energy, cold dark matter, and ordinary matter. The two major ingredients of the standard model, dark matter and dark energy, are far from understood, and their existence presents the most serious challenge to physics since the quantum and relativistic revolutions of a century ago. It is therefore essential to extract as much information as possible from the observations in order to test all aspects of the standard model and to look for possible anomalies that might provide insights into the nature of these two dark components of the cosmos.

In the 1980s successively more stringent limits were placed on the observed level of temperature anisotropy in the CMB (4 5), providing convincing evidence that the dominant matter constituent in the universe is non-baryonic. These searches culminated in the detection of anisotropies by the COBE satellite (6), a confirmation of one of the major predictions of theoretical cosmology (7 $8,9,10,11$ 12). Rapid advances in experimental techniques have since delineated the prominent features in the angular power spectrum (13 $\left.14 \mid \begin{array}{l|l|l|l|}15 & 16 & 17 & 18\end{array}\right)$. The spectrum of fluctuations on large angular scales [low multipole numbers, $l<500$ (19)] has been measured with high precision by the Wilkinson Microwave Anisotropy Probe (WMAP) (20), whereas precise and sensitive CMB observations from the ground and from balloon-based platforms have extended the spectrum to angular scales as small as a few minutes of $\operatorname{arc}(l \approx 3500)(21|22| 23|24| 25)$.

The early universe was opaque to electromagnetic radiation, but as it expanded and cooled the hot electron-baryon plasma combined into neutral hydrogen and helium and the universe became transparent. The microwave background photons that we detect today have passed freely through the universe since they were last scattered by electrons in the ionized plasma. They thus provide a picture of the physical conditions at the time of last scattering when the universe was about 400,000 years old. The angular power spectrum of the $\mathrm{CMB}$ reveals the initial fluctuation spectrum modulated by the effects of acoustic waves in the plasma (26), and it gives quantitative information about the physical conditions in the plasma.

The polarization of the CMB provides an independent way to test the standard model (27 28 [29 30). Anisotropic Thomson scattering of photons at the time of last scattering gives rise to weak linear polarization of the CMB. Measurement of the CMB polarization not only provides an additional way to measure the parameters of the model, it can also verify the correctness of many of the basic assumptions on which the model is founded. It is for this reason that many experiments are being designed to measure the CMB polarization power spectra, despite the difficulty of the observations.

Angular power spectra give the variance $C_{l}$ (usually expressed in terms of CMB temperature, and with units of $\mu \mathrm{K}^{2}$ ) as a function of multipole number $l(26)$. The intensity of polarized radiation can be expressed by the four Stokes parameters $I, Q, U$, and $V$ (31 32). Total intensity is represented by $I$, linear polarization by $Q$ and $U$, and circular polarization by $V$. Thomson scattering does not generate circular polarization, so we ignore $V$. From the other parameters we can generate three power spectra $T T, E E$, and $B B$, where $T$ is the total intensity (Stokes $I$ ) and $E$ and $B$ are the curl-free and curl-like components of the linear polarization field (Stokes $Q$ and $U$ ) (33 34 35) and also three cross-spectra $T E$, 
$T B$, and $E B$. Because of the parity properties of the $T, E$, and $B$ signals, the only non-zero spectra should be $T T, E E, B B$, and $T E$. In the standard model $E$-modes are generated from the primary scalar density fluctuations, whereas $B$-modes are generated only by gravitational-wave tensor fluctuations and secondary processes; the predicted $B B$ power spectrum is undetectable with current sensitivity. A high level of $B B$ would require modifications to the standard model, or it could indicate that the observations are contaminated by radiation from foreground sources, because these are epected to produce both $E$ and $B$ modes in equal measure. The polarization spectrum of the CMB is more difficult to study than the total intensity spectrum because the fractional polarization of the $\mathrm{CMB}$ radiation is no more than $10 \%$. After a number of experiments that placed upper limits on $\mathrm{CMB}$ polarization, $E E$ power has been detected by the DASI [Degree Angular Scale interferometer] experiment (with $6.3 \sigma$ significance) (36 37 38) and the CAPMAP [Cosmic Anisotropy Polarization Mapper] experiment (2.3 $\sigma$ ) (39). TE cross-spectral power has been detected by DASI and by WMAP (40).

The TT spectrum arises from density and temperature fluctuations in the plasma, but polarized radiation, which is caused by the local quadrupole at the time of last scattering, is sensitive to the velocity of the plasma. Because velocity and density are out of phase in an acoustic wave, the maxima in the $E E$ spectrum are out of phase with those in the TT spectra. This phase shift between the spectra is a key feature of the standard model. It has been seen at large angular scales in the TE spectrum by WMAP (41), but it has not yet been verified directly through $E E$ or at the small angular scales corresponding to clusters of galaxies.

We report here observations made with the Cosmic Background Imager (CBI) that have sufficient sensitivity and resolution to detect and measure the second, third, and fourth peaks in the $E E$ spectrum (42), determine the TT-to-EE phase shift, and thus further test the standard model.

\section{The Cosmic Background Imager}

The CBI (Fig. 11 has been making observations of the CMB from a site at $5000 \mathrm{~m}$ elevation on the Chajnantor plateau in the Chilean Andes since late 1999. It is a 13-element radio interferometer receiving radiation in $101-\mathrm{GHz}$ frequency channels covering 26 to $36 \mathrm{GHz}$ (43 44). The individual antennas are $0.9 \mathrm{~m}$ in diameter, and the possible baselines range in length from 1.0 to $5.5 \mathrm{~m}$. An interferometer baseline of length $d$ is sensitive to multipoles $l$ around $2 \pi d / \lambda$ where $\lambda$ is the observing wavelength. The CBI can thus measure the spectrum from $l \sim 300$ to $l \sim 3500$. The antennas are mounted on a platform with azimuth and elevation axes that allow all the antennas to track a point on the sky. The platform can also be rotated about the line of sight; this allows full sampling of all possible baseline orientations and facilitates calibration of the instrumental polarization effects.

Each antenna is sensitive to a single sense of circular polarization, right $(R)$ or left $(L)$. Co-polar baselines, $R R$ and $L L$, are sensitive to Stokes $I \pm V \approx I$ (assuming circular polarization is negligible), whereas cross-polar baselines, $R L$ and $L R$, are sensitive to linear polarization, Stokes $Q \pm i U$ (45). It was thus straightforward to adapt the CBI to measure linear polarization by changing the sense of some of the antennae to maximize the number of cross-polar baselines [Supporting Online Material (SOM) Text].

The observations reported here were carried out between 22 September 2002 and 7 May 2004 using 7 antennae with polarizers set to left circular polarization $(L)$, and 6 antennae with polarizers set to right 
circular polarization $(R)$. To avoid contamination by the sun and moon, observations were made only at night and at angles of greater than $60^{\circ}$ from the moon (supporting online text).

The size of the CBI antennae sets the $l$ resolution of observations made in a single pointing to $\Delta l \approx$ 300 . To obtain the higher resolution in $l$ necessary to resolve the expected structure in the $E E$ spectrum, we had to image a larger area by making a mosaic of overlapping pointings. From 2002 to 2004, we observed a grid of pointings in four regions near the celestial equator that were separated by about 6 hours in right ascension and identified as the $02^{\mathrm{h}}, 08^{\mathrm{h}}, 14^{\mathrm{h}}$, and $20^{\mathrm{h}}$ fields (Fig. 2). These fields were centered on those we observed in 2000 and 2001 in order to measure the $T T$ power spectrum (46 4748 49 50 51 24). The separation of the pointings was 45 arcmin, twice that of the earlier observations, leading to modulation of the sensitivity across the field. For three of the fields we used 36 different pointing positions giving fields $\approx 5^{\circ}$ square, but for the $20^{\mathrm{h}}$ field we divided the available integration time between six pointings in a row; these deeper observations should be more sensitive to any potential systematics.

The largest source of diffuse foreground contamination over the 26- to $36-\mathrm{GHz}$ band is synchrotron radiation from the Galaxy. We chose the four CBI fields, which were constrained to be separated by about 6 hours in right ascension, so as to minimize this contamination (Fig. 3). The CBI $02^{\mathrm{h}}, 08^{\mathrm{h}}$, and $20^{\mathrm{h}}$ fields, like the DASI polarization field, are in regions of low synchrotron emission (Fig. 3), but the $14^{\mathrm{h}}$ field is near the North Polar Spur, and the WMAP observations suggest that this has a higher level of synchrotron foreground. The wide separation of the CBI fields provides some control on foreground contamination, because foreground emission is unlikely to be correlated over such large distances and if it were a problem we would expect to see differences between the spectra of the different fields.

The largest systematic instrumental effect we have to eliminate is ground spillover. Although the ground radiation is unpolarized it enters the CBI feeds after reflection off the inner surface of the shield cans that reduce cross-talk between receivers (43) and therefore gives rise to a highly polarized contaminating signal. This signal is particularly strong on the shortest baselines at the lowest frequencies. To eliminate the ground radiation, we observed sets of six fields separated by $3 \mathrm{~min}$ in right ascension at the same azimuths and elevations, spending $3 \mathrm{~min}$ on each field, so that if the ground emission is constant it should make equal contributions to all six fields. When estimating the power spectrum we made use only of the differences between the fields, ignoring the contribution that is common to all six. This strategy requires that the ground be stable over the total scan duration of 18 minutes, but the penalty is only $\sqrt{6 / 5}$ in flux density sensitivity, equivalent to a factor of 1.2 in observing time.

The data were edited to remove data corrupted by instrumental or atmospheric problems. Amplitude calibration was based on Jupiter (24) and polarization position-angle calibration was based on Tau A, for which we measured a polarization position angle ( $E$ vector) of $-27.6^{\circ}$ by comparison of $\mathrm{CBI}$ and Very Large Array (52) observations of 3C 273 and 3C 279. Instrumental polarization leakage was measured on Tau A and found to be negligible. The noise was estimated from the scatter of the measurements in each 18-minute scan (SOM Text).

After the editing and calibration of the data we made images of the four fields and of all the calibration sources in order to check for possible anomalies (48). As an example, the $I$ image of the $14^{\mathrm{h}}$ field (Fig. (4), made without any subtraction of ground spillover or foreground sources, shows significant power above the level of the noise. This is due to both CMB emission and ground spillover. In the $Q$ and $U$ images (Fig. 4) the signal from the CMB is too weak to identify, and these images are dominated by the regular 
pattern due to ground spillover. The level of the ground spillover in $Q$ and $U$ indicates that there is some ground contamination in the $I$ images as well, although it is somewhat weaker than the CMB signal. When we estimate power spectra, the ground spillover is removed from the data by projecting out (53 21) the common mode in the six matched pointings, so the visibility data set from which the images of Fig. 4 were made is the data which we use in the CMB spectrum determination. But in order to check our procedures we have also made images from the differences of visibilities measured in pointings separated by $9 \mathrm{~min}$ in right ascension; these images should be free of ground contamination (Fig. 5). The total intensity $I$ image shows power well in excess of the noise level, whereas the $Q$ and $U$ images show only noise, the sensitivities per resolution element being too low to reveal the polarization of the CMB. These images also show that leakage of total intensity into the polarization data is small compared to the noise.

\section{Power Spectrum Estimation}

To estimate power spectra from the interferometer visibility measurements we used maximum-likelihood procedures similar to those adopted for earlier experiments (54|37|55). To process the CBI data, we have extended the gridding-based procedure used in our earlier work (49) to deal with mosaicked polarization observations. A given correlator output sample, or visibility, can be one of the four polarization products $R R, R L, L R$, or $L L$. These can be related to the fundamental CMB polarization modes $T$ (temperature), $E$, and $B$ (polarization) (37). The covariances between the measurements depend on the six CMB covariances $T T, E E, B B, T E, T B$, and $E B$. Because the CBI measures circular polarization products, which are orientation independent (depending only on the handedness of the wave polarization), the CBI (or any interferometer using circularly polarized receptors) is sensitive to the $E$ and $B$ modes directly. This simplifies the power spectrum analysis (SOM text).

The principal foreground contamination in total intensity for the $\mathrm{CBI}$ is that due to extragalactic radio sources (47). For the total intensity spectrum, in which discrete sources have a substantial impact, our approach is similar to that used in earlier CBI analyses (47 48 24 49), with minor modifications. Some 3727 NRAO Verly Large Array (VLA) Sky Survey (NVSS) (56) sources with $S_{1.4 \mathrm{GHz}} \geq 3.4 \mathrm{mJy}$ were projected out of the data. In previous work we used separate covariance matrices with different projection factors for sources that were detected at $32 \mathrm{GHz}$ on the OVRO 40-meter telescope and for those that were not. Because in the end there was no gain from this approach, in the present analysis we combined all sources into a single covariance matrix with a single projection factor. For this analysis we assume a uniform variance of $1 \mathrm{Jy}^{2}$ for each source, rather than adjusting the variance for each source. We find that this yields matrices that are numerically more stable under the action of our procedure of completely projecting the source modes out of the data. After a number of tests, we adopted a value of $q_{\mathrm{src}}=100$ for the pre-factor (equivalent to setting the variance on each source flux density to $100 \mathrm{Jy}^{2}$ ).

However, because non-thermal extragalactic radio sources are weakly polarized $(P \leq 10 \%)$ and furthermore only a small fraction of them have $P>2 \%$, only a few of the sources that we projected out in the total intensity spectrum can affect the polarization spectrum. When estimating $E E$ and $B B$, we therefore projected out only a subset of the NVSS sources with $S_{1.4}>3.4 \mathrm{mJy}$. In total 556 of these potentially troublesome point sources need to be considered. These include (i) NVSS sources with $>3 \sigma$ detections of polarized flux density at $1.4 \mathrm{GHz}$ and (ii) sources detected by the $30-\mathrm{GHz}$ OVRO survey of 
the 2000-to-2001 CBI total intensity fields (24). The projection of 556 sources out of the CBI data has only a small effect on the $E E$ power spectrum: in all bins the effect is $\ll 1 \sigma$. In the first two bins, where the polarization detection is strongest, the effect is less than $3 \mu \mathrm{K}^{2}$ for each bin. Both with and without projection, the $B B$ power spectrum is consistent with zero, and the $E E$ spectrum changes very little. Therefore, the sources that we have identified as potentially troublesome (with the criteria described above) have a negligible effect. If we have failed to identify some sources (highly polarized sources just below the NVSS detection limit, for instance), they should add a characteristic $l(l+1) C_{l} \propto l^{2}$ contribution to both $E E$ and $B B$ power spectra, and show up more strongly in the lower frequency channels. No such signature is evident in the CBI data.

We have also studied the effects of point sources using Monte Carlo simulations. To do so we used the NVSS source statistics to characterize the fractional linear polarization of sources, finding a mean $1.4 \mathrm{GHz}$ polarization of $2.7 \%$. Most sources had polarizations less than this; $4 \%$ had polarizations greater than $10 \%$, and $1 \%$ had polarizations greater than $15 \%$. Because the fractional polarization of CMB anisotropies is $\sim 10 \%$, the discrete source foreground will be relatively weaker in polarization than in total intensity. In the $E E$ power spectrum analysis of the simulated data, we find that the first two bins change by less than $4 \mu \mathrm{K}^{2}$ when the source projection is turned on, similar to what is seen in the real CBI data.

The common-mode signal from the ground was removed by constructing a scan covariance matrix assuming a unity correlation between identical visibilities coming from the same scan (e.g., for the six visibilities taken in the consecutive 3 minute integrations that constitute a scan), then passed through the gridding operation. The modes defined by this scan covariance matrix were projected out of the data (53) by applying a large prefactor to this matrix in the likelihood maximization procedure (essentially setting the variance of these modes to be infinite), in the same manner as for the point sources. We used simulated data to determine the best value of the prefactor, and found that too small a value did not completely eliminate the ground spillover, whereas too large a value caused numerical problems. Because there are a large number of sources in the list used for the $T T$ projection, there is an interaction between the source and scan projection matrices $q_{\mathrm{src}} \mathrm{C}^{\mathrm{src}}$ and $q_{\mathrm{scan}} \mathrm{C}^{\mathrm{scan}}$ when the pre-factors $q_{\mathrm{src}}$ and $q_{\mathrm{scan}}$ become large. We explored a range of values for these, and found that for the value of $q_{\mathrm{src}}=100$ a value $q_{\mathrm{scan}}=100$ was in the center of the range for which the $T T$ band powers were stable (there was no significant change in $T T$ amplitude from $q_{\text {scan }}=10$ to $q_{\text {scan }}=100$ ). Similar tests on the real data also showed that the ground signal was eliminated whereas the band powers remained stable.

\section{Polarization Power Spectra}

The CBI measurements for all 10 frequency channels and all four fields observed from 2002 to 2004 have been combined in the maximum likelihood procedure to estimate the $T T, E E, T E$ and $B B$ power spectra (Table 1 and Fig. 6). The scan means (for ground contamination) and point sources have been projected out as described in the previous section. We divided the $l$ range into seven bands, with most of the bins having width $\Delta l=150$. Adjacent bands are anticorrelated at the 10 to $20 \%$ level (fig. 9). Finer binning is possible, but this gives larger band-to-band correlations and is less satisfactory for presenta-

tion. For the quantitative analysis below, we have used bins of width $\Delta l \approx 75$ and taken into account the band-to-band correlations. The results from both binnings are consistent. We have also calculated 
window functions that can be used to calculate the expected band powers in our bands from a theoretical model spectrum (fig. 10).

We compare our results with a fiducial model spectrum (Fig. 6), for which we have chosen the theoretical model (57) with a power law for the primordial spectral index which best fits the first-year WMAP, 2000 CBI, and ACBAR [Arcminute Cosmology Bolometer Array Receiver] CMB total-intensity data [the "WMAPext" data set (1)]. Our results are consistent with the predictions of this model. We have checked this by calculating $\chi^{2}$ for a comparison of our measured band powers and the band powers predicted by the model, with the CBI window functions and the full band-to-band covariance (estimated from the Fisher matrix). The values of $\chi^{2}$ [for 7 degrees of freedom (df)], with the probabilities of obtaining larger values under the null hypothesis in parentheses, are: for $T T, 7.98$ (probability $=0.33$ ); for $E E, 3.77$ (probability $=0.80$ ); for $B B, 4.33$ (probability $=0.74$ ); and for $T E, 5.80$ (probability = $0.56)$.

The TT spectrum shows the same features that we saw in the CBI 2000-to-2001 observations (24), the most prominent being the drop in power between the third and fourth acoustic peaks. The TT spectrum from 2002-2004 is slightly higher than the fiducial model, but the difference is not significant. Both the $E E$ and $T E$ spectra are consistent with the predictions of the fiducial model. The $E E$ spectrum shows detection of power at $l<800$, whereas the $T E$ spectrum is not sufficiently sensitive to show a positive detection. No power is detected in the $B B$ spectrum, as expected on the standard model. The 95\% confidence upper limit on $B B$ power (assuming flat band power in a single $l$ bin) is $7.1 \mu \mathrm{K}^{2}$ with source projection or $2.7 \mu \mathrm{K}^{2}$ without source projection. Because ground radiation and foreground sources are expected to contribute equally to $E E$ and $B B$, this low limit on a possible $B B$ component at multipoles $l<1000$ demonstrates that there is no significant ground or point source contamination at these multipoles and gives confidence in the reliability of the $E E$ spectrum.

At present, the addition of polarization data to CMB $T T$ data has little effect on the values and precision of cosmological parameter estimates, because of the weakness of the polarized signal relative to the total intensity signal. Rather, the strength of the measurement of $E E$ lies in its ability to test a different aspect of the theory. It is nonetheless interesting to explore the effect of the new polarization results on cosmological parameter estimation and to check consistency. As sensitivities improve, future polarization data will have a bigger impact on parameters (58). In this initial investigation including CMB $E E$ polarization data, we explore a limited set of cosmological parameters that has been successful in describing all aspects of CMB data. The model has its basis in the simplest inflationary paradigm, characterized by the following basic set of six parameters: $\omega_{b} \equiv \Omega_{b} h^{2}$, the physical density of baryons; $\omega_{c} \equiv \Omega_{c} h^{2}$, the physical density of cold dark matter; $\theta \equiv 100 l_{s}^{-1}$, parameterizing the angular scale $l_{s}^{-1}$ associated with sound crossing at decoupling, which defines the overall position of the peak-dip pattern; $n_{s}$, the spectral index of the scalar perturbations; $\ln A_{s}$, the logarithm of the overall scalar perturbation amplitude; and $\tau_{c}$, the Thomson scattering depth to decoupling. We do not consider any gravitationalwave induced components because they are not expected to be detectable by the CBI.

The strongest prior we impose is that we only consider flat models $\left(\Omega_{\mathrm{tot}}=1\right)$, as expected in most inflation models. We note that the parameters we derive can change significantly when this prior is relaxed (59 24). We also impose a weak- $h$ prior comprising limitations on the parameter $h(0.45<h<$ 0.90 , where $h \equiv H_{0} /\left(100 \mathrm{~km} \mathrm{~s}^{-1} \mathrm{Mpc}^{-1}\right)$ and $H_{0}$ is the Hubble constant $)$ and on the age of the universe $\left(t_{0}>10 \mathrm{Gyr}\right)$. Within the context of flat models the weak- $h$ prior influences the results very little. We 
do note, however that extreme models with high Thomson depth are excluded by this prior.

In our analysis we consider three combinations of data: (i) WMAP1 only $T T$ and $T E$ results from the first year WMAP data (40), using the likelihood procedure described in (60); (ii) CBI pol plus WMAP1 obtained by adding 14 band powers in each of $T T, T E$, and $E E$ obtained from an analysis of the 2002to-2004 CBI data with a bin width $\Delta l \approx 75$; and (iii) CBI pol plus CBIext plus WMAP1, consisting of all of the CBI polarization data and the addition of high- $l$ bands from our combined mosaic and deep field $T T$ results (24), covering the range $l=600$ to $l=1960$ [bands 5 to 14 of table 1 of (24)]. This third combination extends the data well into the region known as the damping tail, where power is suppressed by photon diffusion and the finite thickness of the last scattering surface.

We use a modified version of the Markov chain Monte Carlo (MCMC) package COSMOMC (61 62) to evaluate probability distributions of the various parameters with respect to the CMB data. We have extended our earlier procedures (24 59 ) to include polarization spectra and the cross correlation between $T T$ and $E E$ spectra (63). In addition to estimating the six cosmological parameters defined above, we determined the distributions of six other derived parameters from the same Markov chains: $\Omega_{\Lambda}$ the energy density in a cosmological constant in units of critical density, the total age of the universe in Gyear; the total energy density of matter, $\Omega_{m}$; the present-day RMS mass fluctuation on $8 h^{-1} \mathrm{Mpc}$ scales, $\sigma_{8}$; the redshift of reionization, $z_{r e}$ (related to $\tau_{c}$ and $\Omega_{b}$ ); and lastly the Hubble parameter $H_{0}$ in units of $\mathrm{km} \mathrm{s}^{-1} \mathrm{Mpc}^{-1} . \Omega_{\Lambda}$ is a derived quantity determined from $\theta$. The amplitude parameter $\sigma_{8}$ is related to $\ln A_{s}$ and has more relevance for comparison with large scale structure data. As expected the inclusion of our polarization results does not have a large impact for this limited parameter set (Table 2). However when including the CBIext $T T$ band powers we obtain significant reduction in the uncertainties, in agreement with (24).

Significance of detection. Our standard CMB power spectrum analysis (64) involves the use of a fiducial $C_{l}$ shape against which the band powers are evaluated. The gridding procedure breaks the power spectrum into top-hat bands in $l$, and thus the (multiplicative) band powers $q_{B}$ effectively break the spectrum into piecewise continous bands that follow the shape $C_{B l}=q_{B} C_{l}$ for $l$ within each band $B$. The most conservative choice for a shape is the flat spectrum $C_{l}=2 \pi / l^{2}$, but one can use a matched shape derived from an actual CMB power spectrum and thus optimally check for deviations from that model. This also allows the use of wider $l$ bands. If we use the fiducial model fitted to the WMAPext dataset (Fig. 6) as our shape, and only project point sources from the TT sector, we find for the CBI data in a single $l$ band a maximum likelihood value band power for $E E$ of $q_{B}=1.22 \pm 0.21$ (68\%) with respect to the WMAP-normalized spectrum, with a value for the log-likelihood with respect to zero of 39.8 (equivalent to an $8.9 \sigma$ detection, where $\sigma=\sqrt{2 \Delta \log \mathcal{L}}$ ). This can be compared with the detection of $6.3 \sigma$ reported for the DASI 3-year results (38). Although there is no indication that the polarization of the foreground point sources is affecting our data, we can also adopt a conservative approach and project out the subset of the brightest sources, as described above. In that case, the best-fit band power $q_{B}$ is $1.18 \pm 0.24(68 \%)$ with log-likelihood with respect to zero of 24.3 (equivalent to $7.0 \sigma$ ). This reduction in significance is due to the increase in uncertainties from the lost modes in this projection, i.e., the drop in band powers is negligible, which again suggests that point sources are not a problem in the $E E$ spectrum. Although we find no evidence for point sources affecting our $E E$ spectrum, we adopt this more conservative value as our estimate of the significance of our detection. 
Phase of the acoustic oscillations. The measurement of the phase of the polarization $E E$ spectrum can, in principle, provide one of the fundamental pillars of the standard model because it tests a unique aspect of the acoustic waves in the photon-baryon fluid. The peak positions in $T T$ are proportional to $\pi l_{s} j$, whereas for $E E$ polarization they are proportional to $\pi l_{s}(j+1 / 2)$, with some corrections from projection effects. To test this, we devised phenomenological models in which the phase-relationship between $T T$ and $E E$ is changed. For these models, we first approximated the fiducial model $E E$ spectrum as a function:

$$
l(l+1) C_{l}^{E E}=f(l)+g(l) \sin (k l+\phi)
$$

where $f$ and $g$ are smooth, non-oscillating functions (we used rational functions with quadratic numerator and denominator) and $k$ is a constant. We then varied $\phi$ to get a range of phase-shifted spectra (Fig. (71A). To determine the goodness-of-fit of the phase-shifted models, we calculated $\chi^{2}$ as a function of the phase $\phi$ and a scaling amplitude $A$, taking into account bin-to-bin correlations using the inverse Fisher matrix (Fig. 7B). For this exercise we used the $\Delta l \approx 75$ binning of the CBI power spectrum. The best-fit phase is $24^{\circ} \pm 33^{\circ}$ with amplitude 0.94 relative to the fiducial model. The fiducial model is well within the $1 \sigma$ (68\%) confidence region (the difference in $\chi^{2}$ between the fiducial model and the best-fit model is 0.64 for $2 \mathrm{df}$ ). The actual data and the best fit model are shown in Fig. $7 \mathrm{C}$. This test shows that our data are entirely consistent with the model predictions, and that we can rule out (at $\approx 3 \sigma$ ) a pathological model in which the $E E$ oscillations are in phase with $T T$ rather than out of phase.

An alternate, and more physically motivated, way to look at the phase of the peaks in $E E$ is to use fits to the fiducial model spectrum of the form

$$
l(l+1) C_{l}^{E E}=\left(A_{s} / A_{s 0}\right)\left(f\left(l \theta / \theta_{0}\right)+g\left(l \theta / \theta_{0}\right) \sin \left(k l \theta / \theta_{0}\right)\right) .
$$

This parameterizes the models in terms of two of the cosmological parameters discussed earlier, $A_{s}$ and $\theta$. The values of these parameters in the fiducial model are $A_{s 0}$ and $\theta_{0}\left(\theta_{0}=1.046\right)$. Changing $\theta$ scales the whole function, including the envelope, rather than just the phase. We now examine the variation of $\chi^{2}$ as these two parameters are changed, the other four cosmological parameters being fixed at their fiducial values. There is a minimum of $\chi^{2}$ near the fiducial model, with $\theta / \theta_{0}=1.02 \pm 0.04$ and $A_{s} / A_{s 0}=0.93$. (A second minimum in which the third polarization peak is shifted and scaled to fit the second fiducial peak is incompatible with the $T T$ data.) This test also shows that the $E E$ data strongly prefer the fiducial model, and demonstrates that the $E E$ data alone have the power to place constraints on cosmological parameters.

Tests for systematics. We have carried out a number of data quality tests to look for possible systematic contamination by foreground emission, residual ground emission, or other instrumental effects. We have found no evidence of significant residual instrumental or foreground effects after correcting for the point sources and projecting out the common ground spillover mode.

Foreground emission is likely to have a different spectrum from the CMB, and ground contamination is frequency-dependent because it depends strongly on the baseline length in wavelengths, and thus shows up most on the shortest baselines at the lowest frequency. To look for these effects we estimated power spectra separately from the data taken in the lower and upper halves of our frequency band, i.e., 26 to 31 and 31 to $36 \mathrm{GHz}$ (fig. 111). We have compared the two spectra by using $\chi^{2}$ (including the bin-to-bin correlations). For $E E$ and $B B$ the measurements are dominated by thermal noise (rather than 
sample variance) so the $\chi^{2}$ results are valid. The results are: $\chi^{2}=8.43(7 \mathrm{df})$ for $E E$, and $\chi^{2}=8.30$ (7 df) for $B B$. The probability of obtaining a larger $\chi^{2}$ by chance is 0.30 for $E E$ and 0.31 for $B B$. The power spectra thus show no indication of strong contamination by foreground emission or residual ground emission. Note that for $T T$ and $T E$ the maximum likelihood error estimates include the effects of sample variance, and, because sample variance is correlated between the two frequency bands, a simple $\chi^{2}$ test is not valid.

In addition to dividing the data into two frequency bands, we carried out jackknife tests in which we compared the following subsets of the data. (i) We compared all subsets of three of the four fields. This would indicate whether any of the fields is anomalous and is a good test for foreground contamination. No significant differences were found, and in particular the $14^{\mathrm{h}}$ field (which lies in the North Polar Spur region) was not anomalous. (ii) We compared all subsets of 12 of the 13 antennae. This would show up problems associated with particular antennae or receivers. (iii) We compared the $T T$ spectra derived from the $R$ and $L$ antennae separately, to check for calibration discrepancies. (iv) We compared spectra estimated from the first and second halves of the data set, to check for effects based on season, distance from the primary calibrator, and other time-dependent parameters. None of these tests showed any significant differences between the data subsets.

The DASI results increase our confidence that diffuse synchrotron emission is not a significant contaminant in our $E E$ spectrum. The fields that we have observed appear to be comparable to the DASI fields (Fig. 3), and the DASI 95\% confidence upper bound of $0.91 \mu \mathrm{K}^{2}$ on $E E$ contamination should also apply to the CBI observations, which were made at higher $l$ where the contribution of synchrotron emission is expected to be lower.

\section{Conclusions}

Our $E E$ results are shown in comparison with the recent results from DASI and CAPMAP in Fig. 8 We have detected the polarized CMB $(E E)$ emission with high confidence $(8.9 \sigma$ when foreground sources are ignored and $7.0 \sigma$ when potentially contaminating sources are projected out), and we have also measured the phase of the $E E$ spectrum and shown that it is consistent with a phase-shift of $\pi$ relative to the $T T$, as expected if acoustic waves are the origin of the features in the $T T$ and $E E$ spectra on the scales of clusters of galaxies. The results from the CBI and DASI experiments are a powerful confirmation of the predictions of the standard model. The CBI continues to observe the polarized CMB emission, and we expect by the end of 2005 to have more than doubled the data set, leading to a decrease of over a factor two in the uncertainties of $C_{l}$.

\section{References and Notes}

1. D. N. Spergel, et al., Astrophys. J. Suppl. 148, 175 (2003).

2. A. G. Riess, et al., Astrophys. J. 607, 665 (2004).

3. M. Tegmark, et al., Phys. Rev. D 69, 103501 (2004).

4. A. C. S. Readhead, et al., Astrophys. J. 346, 566 (1989). 
5. A. C. S. Readhead, C. R. Lawrence, Ann. Rev. Astr. Astrophys. 30, 653 (1992).

6. C. L. Bennett, et al., Astrophys. J. 436, 423 (1994).

7. P. J. E. Peebles, J. T. Yu, Astrophys. J. 162, 815 (1970).

8. R. A. Sunyaev, Y. B. Zeldovich, Astrophys. Space Sci. 7, 3 (1970).

9. J. R. Bond, G. Efstathiou, Mon. Not. R. Astr. Soc. 226, 655 (1987).

10. A. G. Doroshkevich, I. B. Zeldovich, R. A. Siuniaev, Astron. Zh. 55, 913 (1978). Translation: Soviet Astronomy, 22, 523.

11. M. L. Wilson, J. Silk, Astrophys. J. 243, 14 (1981).

12. N. Vittorio, J. Silk, Astrophys. J. 285, L39 (1984).

13. C. B. Netterfield, M. J. Devlin, N. Jarolik, L. Page, E. J. Wollack, Astrophys. J. 474, 47 (1997).

14. S. E. Church, et al., Astrophys. J. 484, 523 (1997).

15. A. D. Miller, et al., Astrophys. J. 524, L1 (1999).

16. E. M. Leitch, et al., Astrophys. J. 532, 37 (2000).

17. P. de Bernardis, et al., Nature 404, 955 (2000).

18. S. Hanany, et al., Astrophys. J. 545, L5 (2000).

19. The power spectrum of a function defined on the celestial sphere is computed by spherical harmonic analysis, in which the multipole number $l$ in analogous to the wavenumber in Fourier analysis. The dipole has $l=1$, and higher $l$ correspond to smaller angular scales, $\theta \approx 180^{\circ} / l(26)$.

20. G. Hinshaw, et al., Astrophys. J. Suppl. 148, 135 (2003).

21. N. W. Halverson, et al., Astrophys. J. 568, 38 (2002).

22. A. Benoit, et al., Astr. Astrophys. 399, L19 (2003).

23. C. Dickinson, et al., Mon. Not. R. Astr. Soc. 353, 732 (2004).

24. A. C. S. Readhead, et al., Astrophys. J. 609, 498 (2004).

25. C. L. Kuo, et al., Astrophys. J. 600, 32 (2004).

26. W. Hu, S. Dodelson, Ann. Rev. Astr. Astrophys. 40, 171 (2002).

27. M. J. Rees, Astrophys. J. 153, L1 (1968).

28. N. Kaiser, Mon. Not. R. Astr. Soc. 202, 1169 (1983).

29. J. R. Bond, G. Efstathiou, Astrophys. J. 285, L45 (1984). 
30. W. Hu, M. White, New Astronomy 2, 323 (1997).

31. G. G. Stokes, Trans. Cambr. Phil. Soc. 9, 399 (1852).

32. S. Chandrasekhar, Radiative Transfer (Clarendon Press, Oxford, 1950).

33. U. Seljak, Astrophys. J. 482, 6 (1997).

34. M. Zaldarriaga, U. Seljak, Phys. Rev. D 55, 1830 (1997).

35. M. Kamionkowski, A. Kosowsky, A. Stebbins, Phys. Rev. D 55, 7368 (1997).

36. E. M. Leitch, et al., Nature 420, 763 (2002).

37. J. M. Kovac, et al., Nature 420, 772 (2002).

38. E. M. Leitch, et al. (2004). Submitted to Astrophys. J., preprint available at http://arXiv.org/abs/astro-ph/0409357

39. D. Barkats, et al. (2004). Submitted to Astrophys. J., preprint available at http://arXiv.org/abs/astro-ph/0409380

40. C. L. Bennett, et al., Astrophys. J. Suppl. 148, 1 (2003).

41. A. Kogut, et al., Astrophys. J. Suppl. 148, 161 (2003).

42. The first peak has very low amplitude and has not yet been detected.

43. S. Padin, et al., Publ. Astron. Soc. Pacific 114, 83 (2002).

44. The CBI Web site is http://www.astro.caltech.edu/ tjp/CBI/

45. W. D. Cotton, in Synthesis Imaging in Radio Astronomy II, vol. 180 of Astronomical Society of the Pacific Conference Series (ASP, San Francisco, 1999), pp. 111-126.

46. S. Padin, et al., Astrophys. J. 549, L1 (2001).

47. B. S. Mason, et al., Astrophys. J. 591, 540 (2003).

48. T. J. Pearson, et al., Astrophys. J. 591, 556 (2003).

49. S. T. Myers, et al., Astrophys. J. 591, 575 (2003).

50. J. L. Sievers, et al., Astrophys. J. 591, 599 (2003).

51. J. R. Bond et al., Astrophys. J. preprint available at http://arXiv.org/abs/astro-ph/0205386

52. The National Radio Astronomy Observatory is a facility of the National Science Foundation operated under cooperative agreement by Associated Universities, Incorporated.

53. J. R. Bond, A. H. Jaffe, L. Knox, Phys. Rev. D 57, 2117 (1998). 
54. M. White, J. E. Carlstrom, M. Dragovan, W. L. Holzapfel, Astrophys. J. 514, 12 (1999).

55. C. Park, K. Ng, C. Park, G. Liu, K. Umetsu, Astrophys. J. 589, 67 (2003).

56. J. J. Condon, et al., Astron. J. 115, 1693 (1998).

57. File wmap_lcdm_pl_model_yr1_v1.txt is available on the LAMBDA web site, http://lambda.gsfc.nasa.gov/.

58. J. R. Bond, C. R. Contaldi, A. M. Lewis, D. Pogosyan, Int. J. Theor. Phys. 43, 597 (2004); available at http://arXiv.org/abs/astro-ph/0406195.

59. J. R. Bond, C. Contaldi, D. Pogosyan, R. Soc. London Philos. Trans. Ser. A 361, 2435 (2003).

60. L. Verde, et al., Astrophys. J. Suppl. 148, 195 (2003).

61. A. Lewis, S. Bridle, Phys. Rev. D 66, 103511 (2002).

62. More information is available at http://cosmologist.info/cosmomc/

63. Our typical run involves the calculation of 16 separate Markov chains. Each chain is run on a separate node of the CITA McKenzie Beowulf cluster for a typical run-time of approximately 3 hours. The chains are run until the largest eigenvalue returned by the Gelman-Rubin convergence test reaches 0.01 .

64. S. T. Myers, in preparation.

65. J. K. Cartwright, A limit on the polarization of the cosmic microwave background radiation, Ph.D. thesis, California Institute of Technology (2002).

66. J. K. Cartwright, et al., Astrophys. J. (2004). In preparation.

67. J. M. Kovac, Detection of polarization in the cosmic microwave background using DASI, Ph.D. thesis, University of Chicago (2003).

68. The VLA measurements are available at http://www.vla.nrao.edu/astro/calib/polar/

69. J. Dubinski, R. Humble, U.-L. Pen, C. Loken, P. Martin, High performance commodity networking in a 512-cpu teraflop beowulf cluster for computational astrophysics (2003). Preprint available at http://arXiv.org/abs/astro-ph/0305109

70. L. S. Blackford, et al., ScaLAPACK Users' Guide (Society for Industrial and Applied Mathematics, Philadelphia, PA, 1997).

71. J. L. Sievers, Data analysis of and results from observations of the cosmic microwave background with the Cosmic Background Imager, Ph.D. thesis, California Institute of Technology (2003).

72. C. L. Bennett, et al., Astrophys. J. Suppl. 148, 97 (2003). 
73. We gratefully acknowledge the generous support of the Kavli Operating Institute, which made the polarization upgrade possible, and thank B. and S. Rawn, Jr., for their continuing support. We are also grateful for the support of M. and R. Linde, C. and S. Drinkward, and the provost, president, and PMA division chairman of the California Institute of Technology. This work was supported by the NSF under grants AST 9413935, 9802989, 0098734, and 0206416. The computing facilities at CITA were funded by the Canada Foundation for Innovation. JRB, CRC, JLS, and DP acknowledge support from the Canadian Institute for Advanced Research and Natural Science and Engineering Research Council at CITA. SC acknowledges support by Fondo Nacional de Deasrollo Cientifico y Tecnológico(FONDECYT) grant 1030805. RB was supported partially by Comisión Nacional de Investigación Cientifica y Tecnológica (CONICYT). LB, SC, and JM acknowledge support from the Chilean Center for Astrophysics Programa Fondo de Investigación Avanzada en Areas Prioritarias (FONDAP) No. 15010003. ST acknowledges support by grant Milenio ICM P02-049. JEC, JKC, EML, and CP acknowledge support from NSF grant OPP-0094541 to the Kavli Institute for Cosmological Physics, an NSF Physics Frontier Center. We thank CONICYT for granting permission to operate within the Chanjnantor Scientific Preserve in Chile, and the National Radio Astronomy Observatory (NRAO) Central Development Lab for developing the High Electron Moblity Transistor amplifiers used in this project and assisting with production. We thank N. Oyarce, W. Araya, and J. Cortes for their dedicated work in operating the CBI.

\section{Supporting Material}

Modifications for polarization measurement The polarization observations reported in this paper were made between September 2002 and May 2004. Earlier tests of the technique were made using a single cross-polarized antenna (65) 66). The CBI was upgraded in 2002 to enhance its polarization capability. This involved replacement of the existing polarizers with new broadband achromatic polarizers, replacement of the high-electron-mobility-transistor (HEMT) amplifiers with new lower-noise amplifiers, and reconfiguration of the antennae into a more compact array.

The circular polarization mode ( $R$ or $L$ ) received by each antenna can be selected by changing the orientation of a quarter-wave plate in front of the low noise amplifier. The original CBI quarter-wave plates were replaced by achromatic DASI-style polarizers (67) 36) that could be rotated under computer control so that the polarization in any antenna can be changed in $<5 \mathrm{~s}$. An important design goal for polarization observations is to limit the polarization impurity: if an antenna does not receive pure $R$ or $L$ polarization the linear polarization measurements will be corrupted by an admixture of total intensity $I$. The fraction of $I$ that appears in the $R L$ or $L R$ visibility measurement is called the leakage. This is a complex number (amplitude and phase) that must be measured for each antenna. The new polarizers reduced the leakages from $\sim 5-15 \%$ to $\sim 1-3 \%$. The leakages are stable, and typically exhibit changes of $<0.2 \%$ over periods of a few months.

In the first two years of operation of the CBI (2000 and 2001) we used sparse configurations of the antennae in order to cover a wide range of multipoles $(300<l<3500)$. For the polarization observations we decided to concentrate on the multipole range 300-2000 in order to provide maximum sensitivity in the region where the CMB polarized $E E$ signal is expected to be the greatest. For this reason we adopted the close-packed configuration shown in Fig.11This configuration provides the highest 
concentration of short baselines possible with the CBI and provides an excellent match to the $l$ range of the expected maximum $E E$ signal.

Data calibration and editing. During the observations and initial data analysis, we inspected each night's observations to look for instrumental and other problems. For the final analysis, we used automatic procedures to remove data with known problems (warm or unstable receivers, for example) and with higher than normal noise levels. This last check eliminated $\approx 1 \%$ of the data that had been corrupted by clouds or instrumental problems.

Amplitude calibration. For the CBI, the amplitude and phase calibration of the co-polar visibility data ( $R R$ or $L L$ ) was carried out using the same procedures as for the 2000-2001 observations (47). The refinement of the CBI flux density scale using the WMAP observations of Jupiter has been described in (24). The uncertainty in the revised scale is $1.3 \%$ in flux density (2.6\% in the power spectrum, $C_{l}$ ). On most nights one or more of the primary calibration sources Tau A (the Crab Nebula), Jupiter, Saturn, and 3C 274 was observed. All of these gave consistent results, except for 3C 274: we found that the flux density of 3C 274 declined by $7 \%$ over the period of these observations. The model for 3C 274 was adjusted to take this secular variation into account before the final calibration of the data.

The majority of CBI data are calibrated using measurements of Tau A and Jupiter, as in (24). When none of the primary calibration sources was available we used secondary calibration sources, such as the variable quasar J1924-293, for which we obtained flux densities by interpolating from adjacent days calibrated against the primary calibrators.

Polarization calibration. The calibration measurements on the co-polar baselines yield complex gain factors for each antenna. These gain factors are sufficient to calibrate the cross-polarized baselines ( $L R$ and $R L$ ) except for an unknown phase difference between the $R$ antennae and the $L$ antennae, equivalent to an unknown rotation of the plane of linear polarization (36 45). We determine the unknown $L-R$ phase difference by observations of a strong, polarized calibration source, Tau A, for which we assume the polarization position angle ( $E$-vector) is $-27.6^{\circ}$. This value was derived $(6566)$ by comparison of CBI and Very Large Array (52) observations of 3C 273 and 3C 279, both of which vary but are observed regularly with the CBI at $26-36 \mathrm{GHz}$ and the VLA at frequencies straddling the CBI band $(22 \mathrm{GHz}$ and $44 \mathrm{GHz}$ (68). It is close to the angle measured with other instruments at lower frequencies. The $L-R$ phase difference is very stable unless receivers or cables are modified, so on nights when no measurement of Tau A was available we used the average of all the Tau A measurements.

Leakage measurement We measured the instrumental polarization leakage factors on each night when either Tau A or Jupiter could be observed. These observations were made at a number of different parallactic angles, by rotating the CBI platform, to enable the source and instrumental polarization to be separated. The instrumental leakage was found to be in the range $1 \%-3 \%$ on most baseline-channels, with a few baseline-channels showing leakages as high as 5\%. We determined that leakage did not vary significantly across the field of view by making observations of Tau A at a number of offset positions. This low level of instrumental polarization and our strategy of rotating the deck so that many antenna 
pairs contribute to the same $(u, v)$ point ensures that any instrumental polarization in our final data set is negligible $(<1 \%)$. We have therefore ignored the instrumental polarization in the present analysis.

Noise calculation. In order to ensure that the ground contamination was identical in each of a set of six pointings, we deleted all visibility samples that did not have counterparts observed at the same hour angle (within the tolerance of the integration time, $4.2 \mathrm{~s}$ ) in all of the six fields. After selecting matched data points in this way, we calculated the noise from the scatter of the visibility measurements. As an error in the noise estimate will bias the final power spectrum estimate, it is important to obtain an accurate estimate of the noise in the data. In one scan, comprising observations of 6 fields, we record $m=1, \ldots, M$ ( $M$ varies, but is usually about 37) data points (complex visibilities) for each of $n=1, \ldots, N$ fields $(N=6)$. The observed visibility $V_{n m}$ is related to the true visibility $X_{n}$ and the ground contribution $g_{m}$ by

$$
V_{n m}=X_{n}+g_{m}+r_{n m}
$$

where $r_{n m}$ is the noise in the measurement. Note that $X_{n}$ is the same for all $m$ (we do not change the baseline length or orientation relative to the sky during the scan), and $g_{m}$ is the same for all $n$ (the ground contribution is assumed to be the same in each field, i.e., constant for the duration of the scan at a given elevation and azimuth). An estimator for the noise variance is

$$
\frac{1}{(M-1)(N-1)} \sum_{n=1}^{N} \sum_{m=1}^{M} \Psi_{n m}^{2} .
$$

where $\Psi_{n m}$ is derived from $V_{n m}$ by subtracting the mean of the $N$ measurements from all $N$ obtained at each time $m$, and the mean of the $M$ measurements from all $M$ obtained on each field $n$. Our best estimator is the average of the two estimates obtained by treating the real and imaginary parts of the visibility separately. We obtained a single noise estimate for each baseline-channel that applies to a whole 18 min scan. The variance of the estimator is

$$
\operatorname{Var}\left(\widehat{\sigma^{2}}\right)=\frac{\sigma^{2}}{(M-1)(N-1)}
$$

The uncertainty in the noise estimate in each scan is small enough that noise bias (47) is not a concern in the present observations. Scans with rms noise more than three times that expected for normal system temperatures were deleted; in most cases the high noise was due to clouds.

Power spectrum estimation The principles of estimating polarization power spectra from interferometer visibility measurements are described by (37). To process the CBI data, we have extended the gridding-based procedure used in our earlier work (49) to deal with mosaicked polarization observations. A given correlator output sample, or visibility, can be one of the four polarization products $R R, R L, L R$, or $L L$. These can be related to the fundamental CMB polarization modes $T$ (temperature), $E$, and $B$ (polarization) through the expressions given in Equations 3 and 4 of (37). The resulting power spectra are decomposed into the six possible covariances $T T, E E, B B, T E, T B$, and $E B$. Note that because the CBI measures circular polarization products, which are orientation independent (depending only on the handedness of the wave polarization), the CBI (or any interferometer using circularly polarized receptors) is sensitive to the $E$ and $B$ modes directly. This simplifies the power spectrum analysis. 
The co-polar $R R$ and $L L$ visibilities are gridded together into an effective $R R$ estimator $(\langle L L\rangle$ and $\langle R R\rangle$ are identical in the absence of circular polarization) as in (49), while the cross-polar $R L$ and $L R$ visibilities are gridded together, after conjugating and reflecting the $L R$ visibilities in the $u v$ plane, into cross-polar estimators $\boldsymbol{\Delta}_{R L}$ using the same gridding kernel as the co-polar data. The covariance matrix elements are computed for the cross-polar estimators using a modified operator $\mathbf{P}_{R L}(\boldsymbol{v})=$ $\mathbf{P}(\boldsymbol{v}) e^{i 2(\chi-\psi)}$ where $\mathbf{P}$ is defined in Equation 12 of (49), $\psi$ is the on-sky parallactic angle of the CBI receivers (Equation 2 of (36) and $\chi$ is the wave-vector angle corresponding to the $u v$ point $\boldsymbol{v}$ (Equation 3 of (37)). The band powers derived from the likelihood analysis are then $\left\{q_{B}^{\mathrm{S}}, B=1, \ldots, N_{B}^{\mathrm{S}}\right\}$; the different covariance products $S=T T, E E, B B, T E, T B, E B$ can have different numbers and locations of bands. Point sources are handled in the same manner as in (49), with the option of projecting out the sources from the $R L$ and $L R$ parts of the covariance. The new scanning procedure required the addition of a scan projection matrix $\mathbf{C}^{\text {scan }}$ constructed by building a "noiselike" matrix as in Equations 32 and 35 of (49), with the covariance elements $E_{k k^{\prime}}=1$ if visibilities $k$ and $k^{\prime}$ are from the same scan (otherwise zero); this is then projected out with a pre-factor $q_{\text {scan }}$ in the same way as the point sources are. Details of this modified procedure will be given in (64).

The maximum likelihood estimation of the spectrum from the gridded estimators is done on the CITA McKenzie cluster (69) which consists of 256 nodes with two $2.4 \mathrm{GHz}$ Intel Xeon processors and $1 \mathrm{~GB}$ of memory per node. The matrix operations are done using the SCALAPACK library (70). From an initial guess of the spectrum, we iterate to the maximum likelihood solution using the Newton-Raphson method. One modification to the procedure used in (53) provides a significant improvement. Rather than use the standard approximation to the curvature, with which the number of expensive matrix operations is proportional to the number of bins in the spectrum, we use an approximate curvature that requires only a single matrix inversion (71). Using 32 nodes per mosaic, with $10^{4}$ estimators per mosaic, this decreases the time per iteration from about an hour to one minute, without changing the solutions. The total time for the spectrum to converge, once the estimators and correlations are read into memory, is about 10 minutes, and is virtually independent of the number of bins in the spectrum. 
Table 1: CBI Band Powers in $\mu \mathrm{K}^{2}$. The broad first band is not sensitive over the $0-600$ range, but with finer binning we have a detection in a band near 400 .

\begin{tabular}{|c|c|c|c|c|c|c|c|c|c|}
\hline & & $\mathrm{TT}$ & & $\mathrm{EE}$ & & BB & & $\mathrm{TE}$ & \\
\hline $1-r$ & nge & Power & Error & Power & Error & Power & Error & Power & Error \\
\hline 0 & 600 & 2882.0 & 276.8 & 13.7 & 6.5 & -0.7 & 5.5 & 8.7 & 32.1 \\
\hline 600 & 750 & 1865.8 & 275.8 & 38.9 & 11.6 & 7.2 & 8.1 & -15.6 & 41.0 \\
\hline 750 & 900 & 2413.4 & 342.7 & 2.4 & 17.9 & -1.1 & 16.7 & 17.9 & 57.6 \\
\hline 900 & 1050 & 1098.0 & 306.5 & 54.8 & 33.5 & 14.6 & 30.8 & -96.7 & 76.6 \\
\hline 1050 & 1200 & 1411.4 & 303.6 & -22.1 & 26.8 & 20.8 & 30.7 & -57.1 & 67.1 \\
\hline 1200 & 1500 & 988.9 & 189.1 & 21.6 & 25.9 & 23.6 & 26.9 & -49.6 & 54.2 \\
\hline 1500 & 5000 & 191.4 & 164.2 & -36.8 & 55.7 & -55.5 & 51.8 & 29.4 & 91.4 \\
\hline
\end{tabular}

Table 2: Cosmological constraints from the WMAP1 only, CBI pol plus WMAP1, and CBI pol plus CBIext plus WMAP1 data compilations for an assumed $\Omega_{t o t}=1$.0. Relaxation of this constraint opens up the tight uncertainties on $H_{0}$ and $\Omega_{m}$. We included weak external priors on the Hubble parameter $\left(45 \mathrm{~km} \mathrm{~s}^{-1} \mathrm{Mpc}^{-1}<H_{0}<90 \mathrm{~km} \mathrm{~s}^{-1} \mathrm{Mpc}^{-1}\right.$ ) and the age of the universe $\left(t_{0}>10\right.$ Gyear). The flatness prior has the strongest effect on the parameters by breaking the geometrical degeneracy and allowing us to derive tight constraints on $H_{0}$ and $\Omega_{m}$. The top six parameters are those used in the Markov chain evaluations and the distributions of the bottom six are derived from the same chains. The uncertainties are given as $68 \%$ confidence intervals.

\begin{tabular}{c|ccc}
\hline & WMAP1 & CBIpol+WMAP1 & CBIpol+CBIext+WMAP1 \\
& & & \\
\hline & & & \\
$\Omega_{b} h^{2}$ & $0.0243_{-0.0017}^{+0.0019}$ & $0.0240_{-0.0016}^{+0.0018}$ & $0.0233_{-0.0013}^{+0.0013}$ \\
$\Omega_{c} h^{2}$ & $0.119_{-0.016}^{+0.016}$ & $0.113_{-0.015}^{+0.014}$ & $0.109_{-0.013}^{+0.012}$ \\
$\theta$ & $1.049_{-0.008}^{+0.007}$ & $1.048_{-0.006}^{+0.006}$ & $1.044_{-0.005}^{+0.005}$ \\
$\tau_{c}$ & $0.188_{-0.065}^{+0.037}$ & $0.190_{-0.067}^{+0.044}$ & $0.164_{-0.053}^{+0.027}$ \\
$n_{s}$ & $1.01_{-0.05}^{+0.06}$ & $1.00_{-0.05}^{+0.06}$ & $0.98_{-0.04}^{+0.04}$ \\
$\log \left[10^{10} A_{S}\right]$ & $3.3_{-0.2}^{+0.2}$ & $3.3_{-0.2}^{+0.2}$ & $3.2_{-0.2}^{+0.2}$ \\
$\Omega_{\Lambda}$ & $0.72_{-0.07}^{+0.08}$ & $0.74_{-0.07}^{+0.07}$ & $0.75_{-0.06}^{+0.06}$ \\
$\mathrm{Age}(\mathrm{Gyr})$ & $13.3_{-0.4}^{+0.4}$ & $13.4_{-0.4}^{+0.3}$ & $13.5_{-0.3}^{+0.3}$ \\
$\Omega_{m}$ & $0.28_{-0.08}^{+0.07}$ & $0.26_{-0.07}^{+0.07}$ & $0.25_{-0.06}^{+0.06}$ \\
$\sigma_{8}$ & $0.94_{-0.13}^{+0.13}$ & $0.91_{-0.10}^{+0.10}$ & $0.85_{-0.08}^{+0.08}$ \\
$z_{r e}$ & $17.5_{-6.2}^{+6.7}$ & $17.5_{-6.2}^{+6.7}$ & $16.0_{-5.5}^{+6.0}$ \\
$H_{0}$ & $73.3_{-6.4}^{+7.1}$ & $74.5_{-6.5}^{+7.7}$ & $74.2_{-5.5}^{+6.1}$ \\
\hline
\end{tabular}




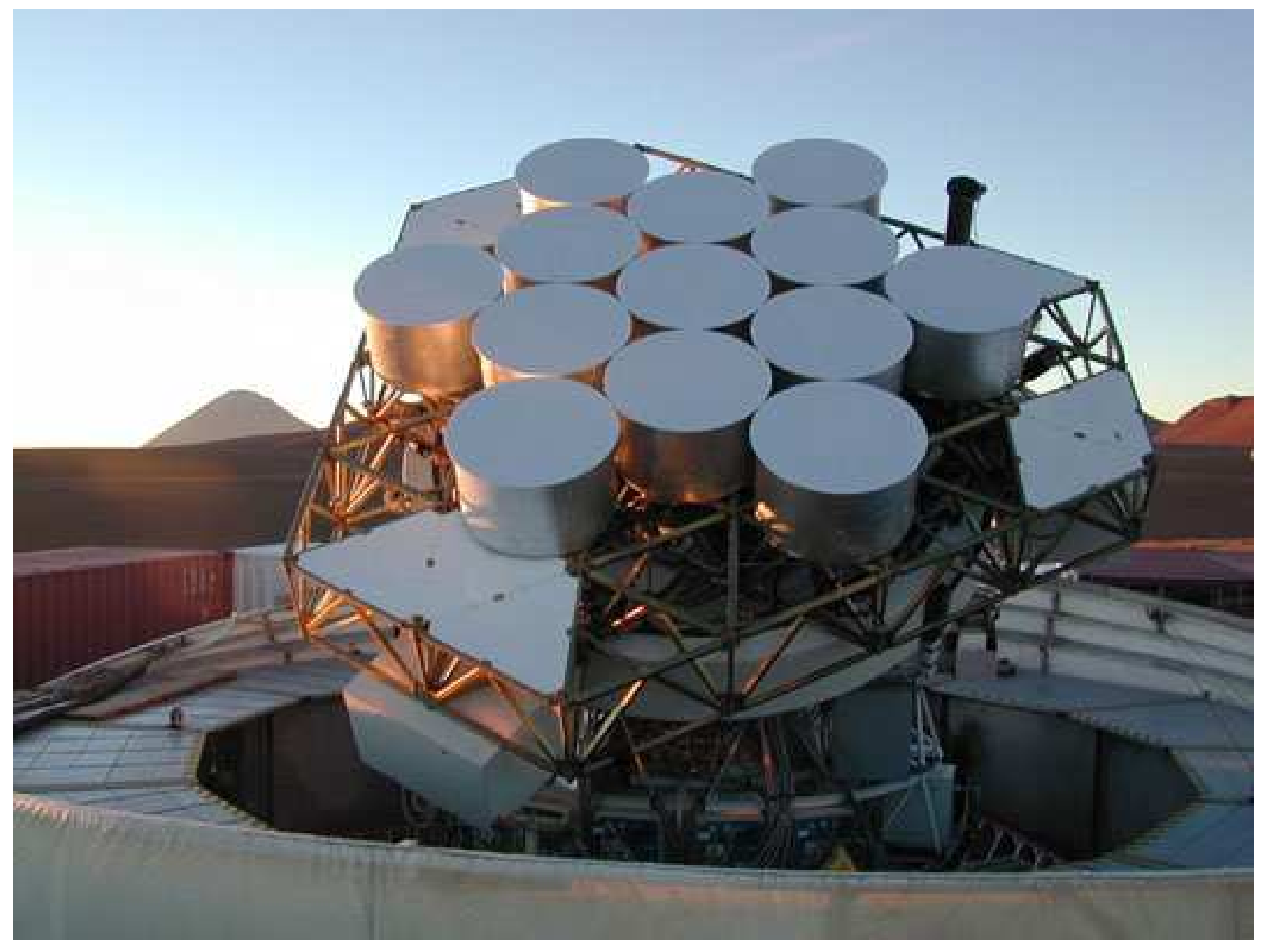

Figure 1: The Cosmic Background Imager. For the polarization observations, the 13 90-cm Cassegrain antennae were arranged in this hexagonal close-packed configuration on the rotating, alt-az mounted platform, with six adjusted to be sensitive to right-hand circularly polarized radiation and seven to left-hand circularly polarized radiation. By correlating the signals from the antennae in pairs, 78 interferometer baselines are obtained ranging in length from 1.0 to $3.5 \mathrm{~m}$. 

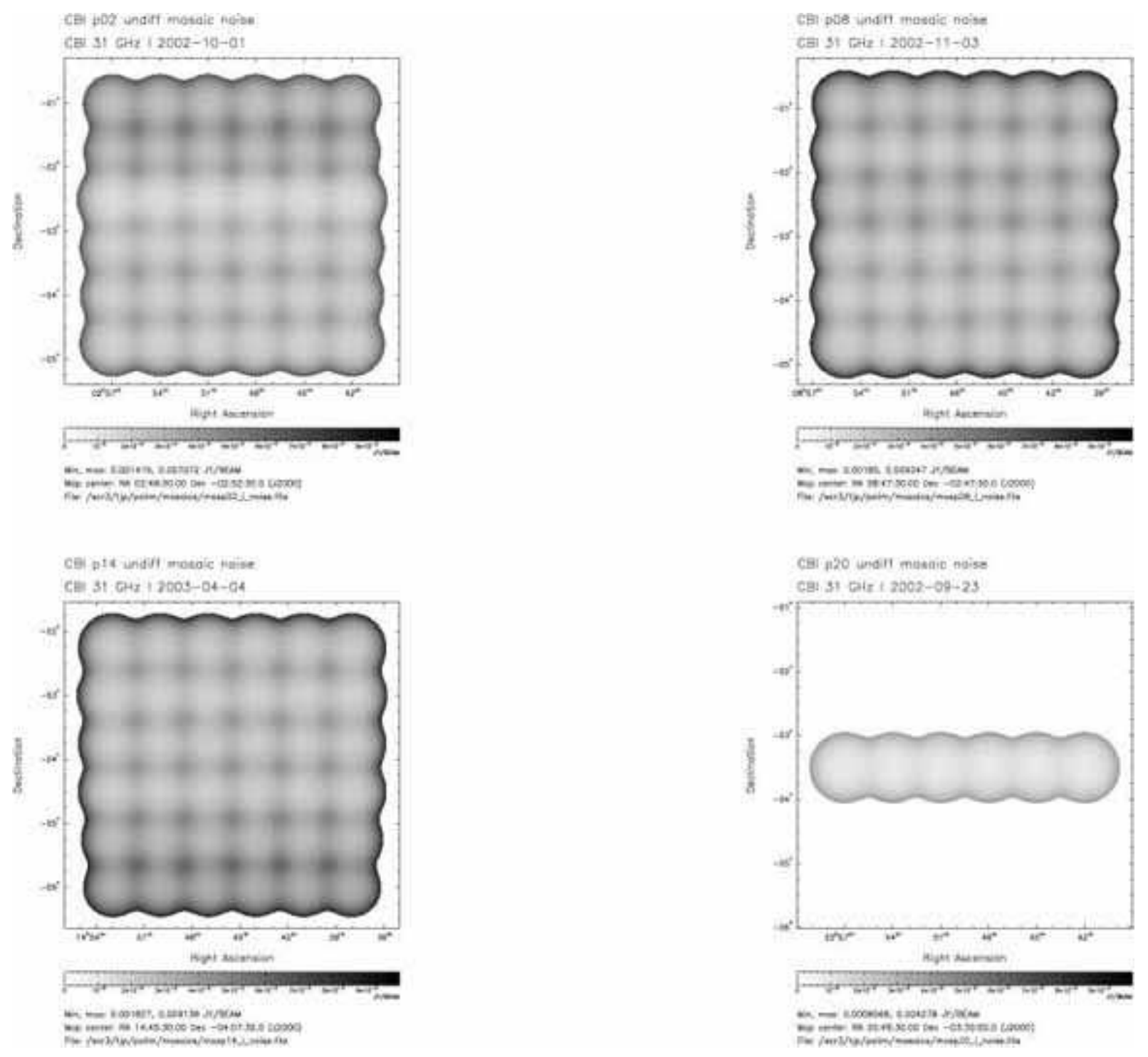

Figure 2: The sky coverage of the four fields imaged by the CBI in polarization. The grey-scale shows the noise-level achieved in total intensity, $I$, in the observations reported here. Three of the fields have been mapped with 36 separate pointings, whereas the fourth, $20^{\mathrm{h}}$, has been mapped more deeply but in only six pointings. The modulation of the sensitivity by the CBI primary beam is apparent. The approximate centers of the four fields are: $02^{\mathrm{h}} 49^{\mathrm{m}} 30^{\mathrm{s}},-02^{\circ} 52^{\prime} 30^{\prime \prime} ; 08^{\mathrm{h}} 47^{\mathrm{m}} 30^{\mathrm{s}},-02^{\circ} 47^{\prime} 30^{\prime \prime} ; 14^{\mathrm{h}} 45^{\mathrm{m}} 30^{\mathrm{s}}$, $-04^{\circ} 07^{\prime} 30^{\prime \prime} ; 20^{\mathrm{h}} 49^{\mathrm{m}} 30^{\mathrm{s}},-03^{\circ} 30^{\prime} 00^{\prime \prime}$ (J2000 right ascension and declination). 


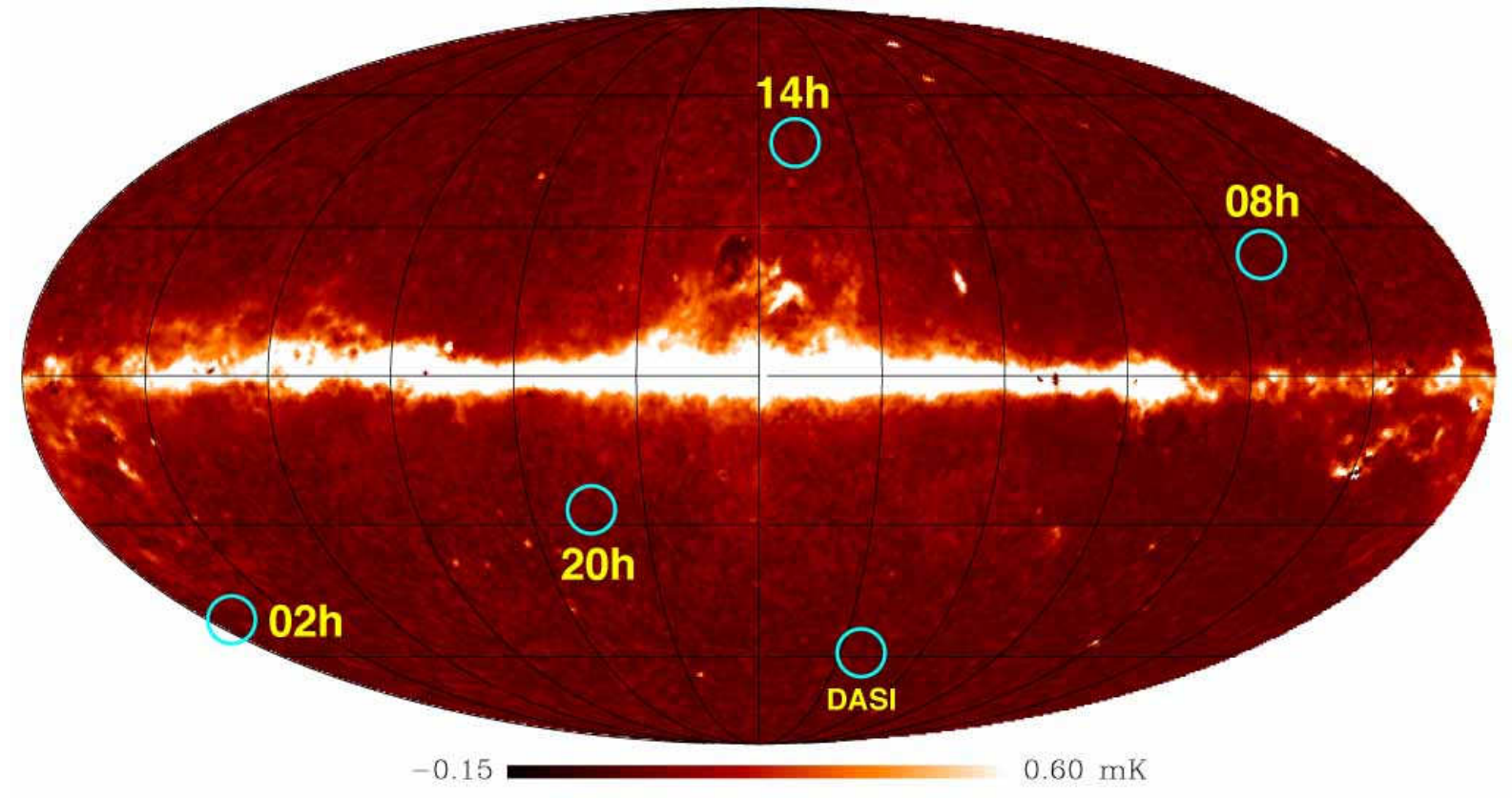

Figure 3: Location of CBI and DASI fields in relation to the Galaxy. The sky image is the Ka-band synchrotron map derived from WMAP first year data (72). Galactic longitude increases to the left, with zero in the center of the image. 

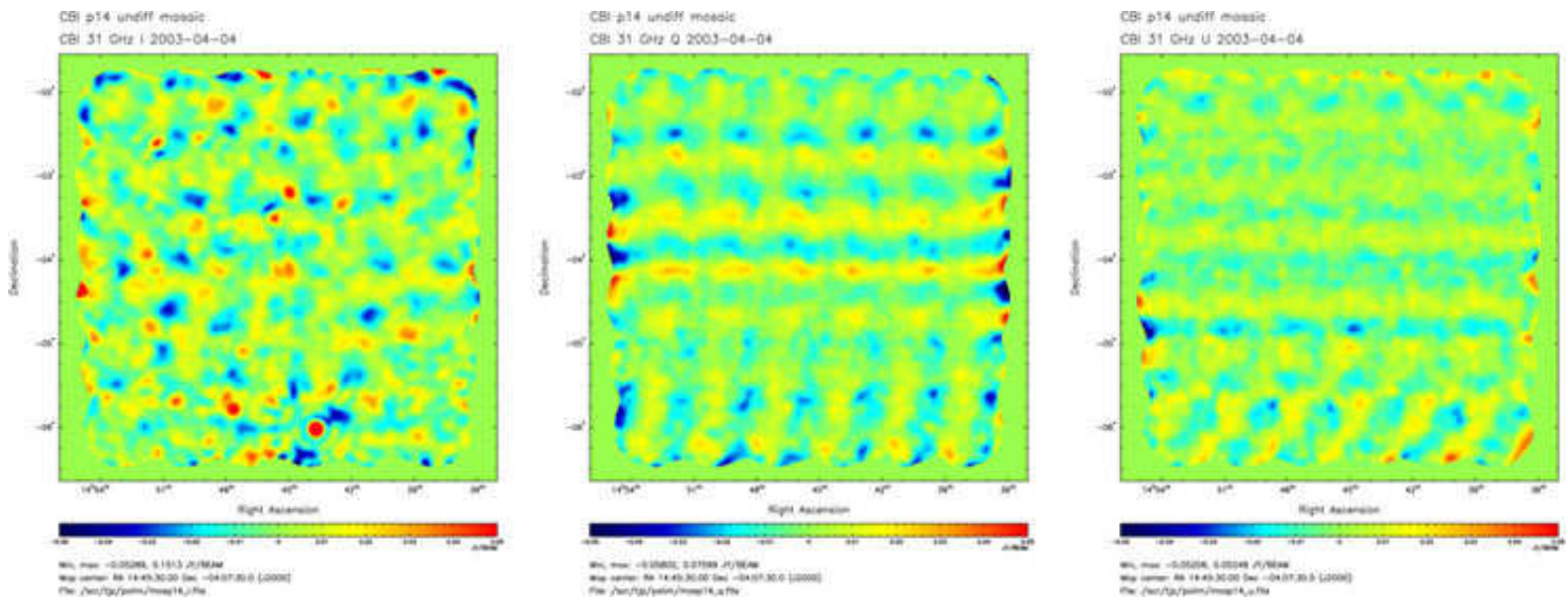

Figure 4: Images of the $14^{\mathrm{h}}$ field mapped by the CBI in Stokes parameters $I, Q$, and $U$ (Stokes $V$, circular polarization, is not measured and is assumed to be zero). Color is used to represent intensity, with the same scale in each Stokes parameter. In these images the contaminating effects of ground radiation and foreground emission have not been removed. The total intensity, $I$, image (left) is dominated by CMB emission (modulated by the instrumental point-spread function); some foreground point sources are visible (red spots). The linear polarization, $Q$ and $U$, images (center and right) are dominated by instrumental noise and ground pickup. Ground pickup, which with our observing strategy should be the same in each pointing at the same declination, gives rise to a pattern that repeats at intervals of 3 min in right ascension. 

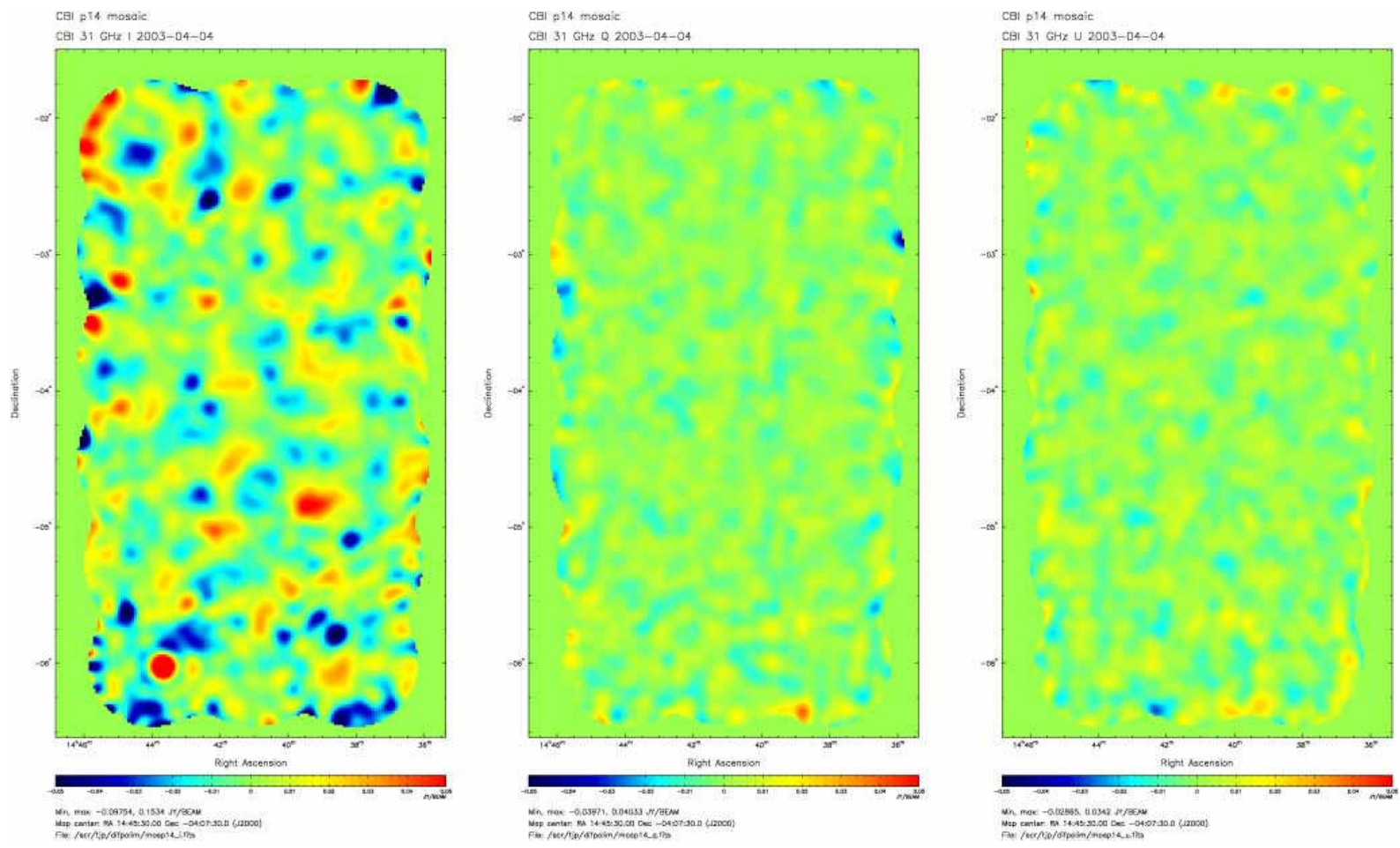

Figure 5: The effect of lead minus trail differencing. Here the data presented in Fig. 4have been differenced: each visibility measurement has had the corresponding measurement on a field 9 min later in right ascension subtracted. Because the ground pickup is very similar for both measurements, ground emission cancels out in the difference. In the resulting images foreground point sources may appear positive or negative in $I$ depending on their right ascension. The $Q$ and $U$ images show that ground pickup has been removed with high accuracy. 

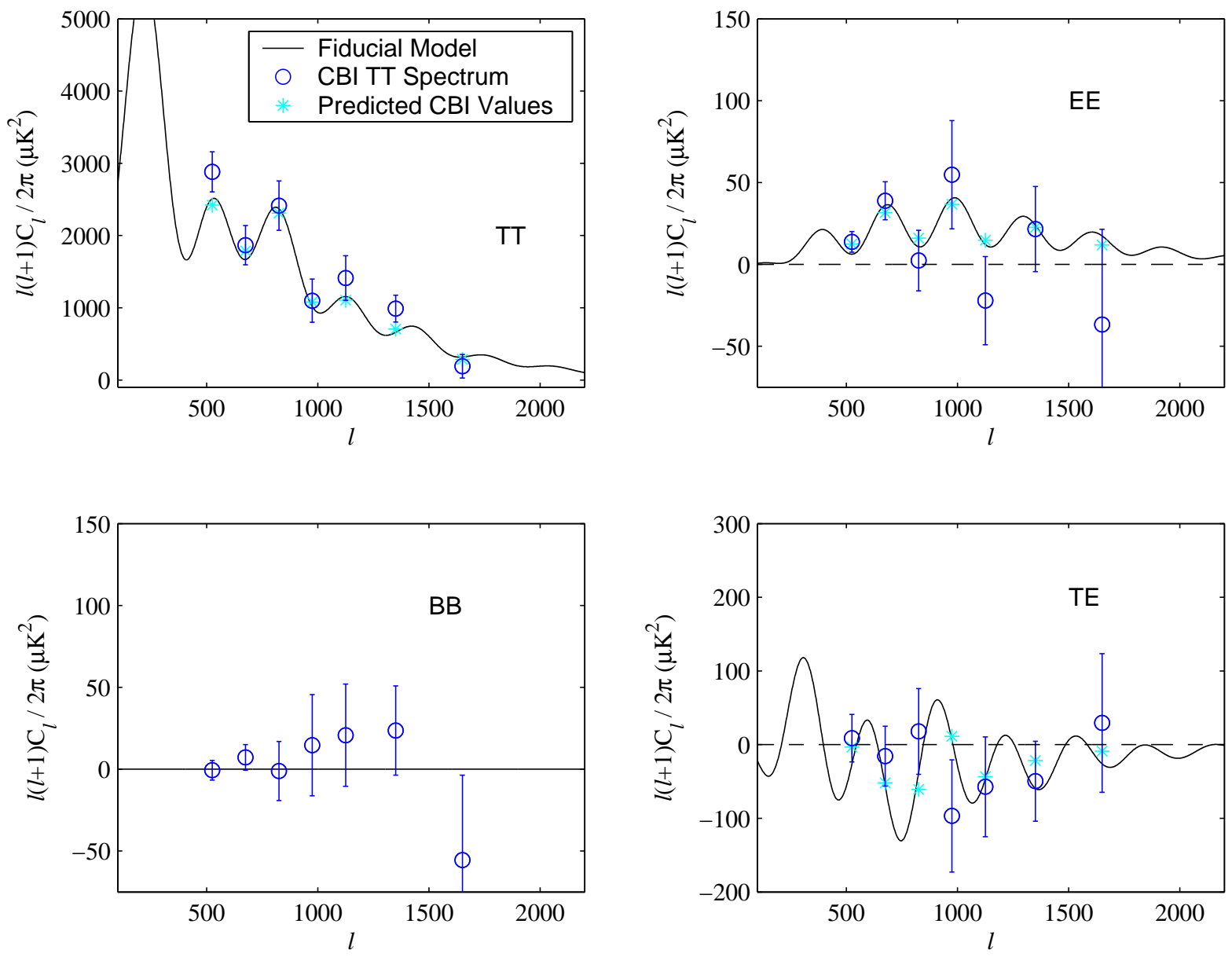

Figure 6: Power spectra of CMB polarization from the CBI measurements. The four panels show total intensity power spectrum $T T$, grad polarization mode power spectrum $E E$, curl polarization mode power spectrum $B B$, and cross-spectrum $T E$. Numerical values are given in Table 1 The black curve is the theoretical $\Lambda \mathrm{CDM}$ model using a power law for the primordial spectral index which best fits the WMAP, CBI, and ACBAR CMB data (57). The predictions of this model for the CBI bands using the CBI window functions are indicated by the stars. 

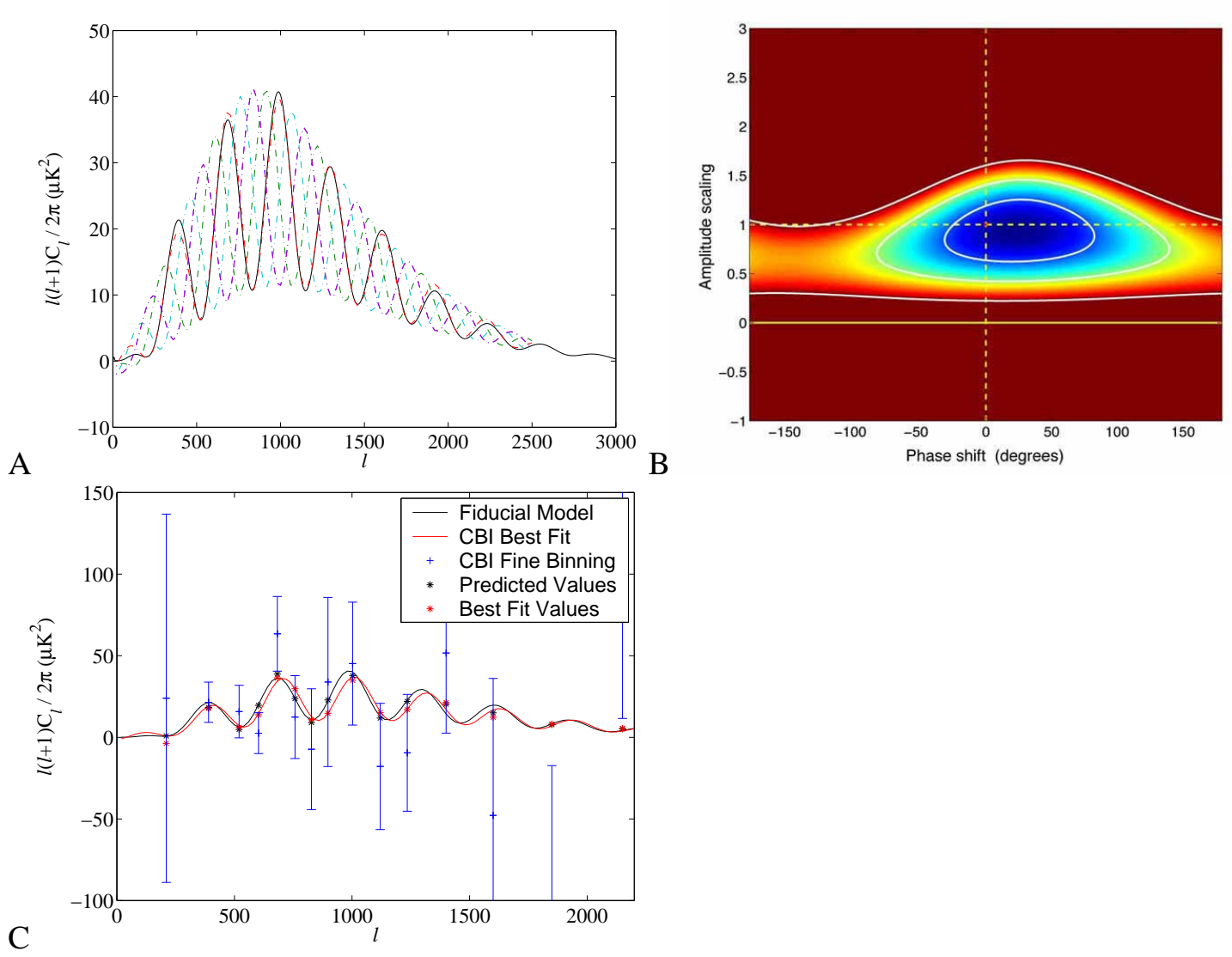
B

Figure 7: Sensitivity of the CBI $E E$ power spectrum to the phase of the acoustic oscillations. (A) The $E E$ spectrum predicted by the fiducial model (black line) with a variety of "phase shifted" spectra with similar envelopes calculated as described in the text. (B) Goodness-of-fit $\left(\chi^{2}\right)$ for the model as a function of two parameters: the phase shift (horizontal axis) and an overall scaling (vertical axis); the point at $(0,1)$ indicates the fiducial model corresponding to a standard $\Lambda \mathrm{CDM}$ model. Contours are at 1, 2, and $3 \sigma$ intervals (i.e., $\Delta \chi^{2}=2.30,6.17$, and 11.8 for $2 \mathrm{df}$ ). (C) Comparison of the fit of the fiducial model (black line) and the minimum- $\chi^{2}$ phase-shifted model (red line) with the CBI data points (blue) and the band powers predicted by the models (black and red stars). 


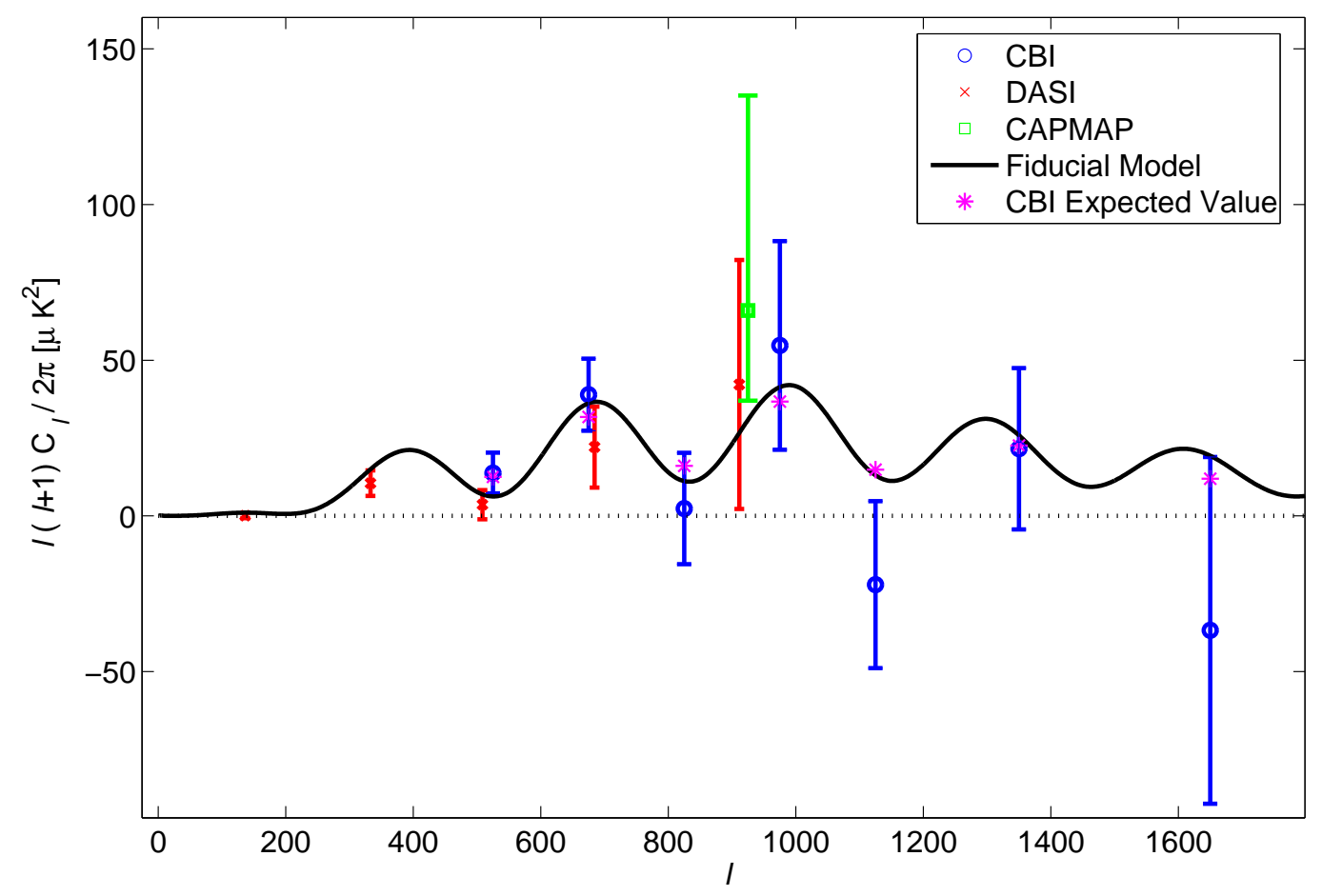

Figure 8: Comparison of $E E$ measurements from CBI, DASI (38), and CAPMAP (39). The fiducial model curve is the same as in Fig. 6 . The asterisks show the predictions of the fiducial model for the CBI bands.

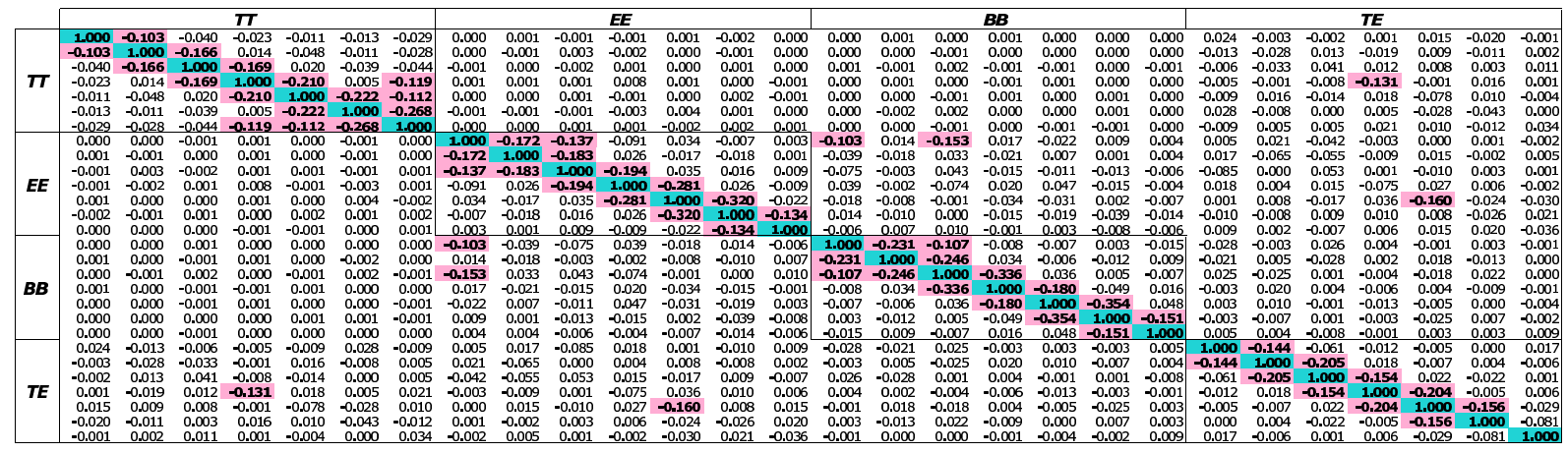

Figure 9: Normalized band-to-band correlations for the 7 bands shown in Table 1 

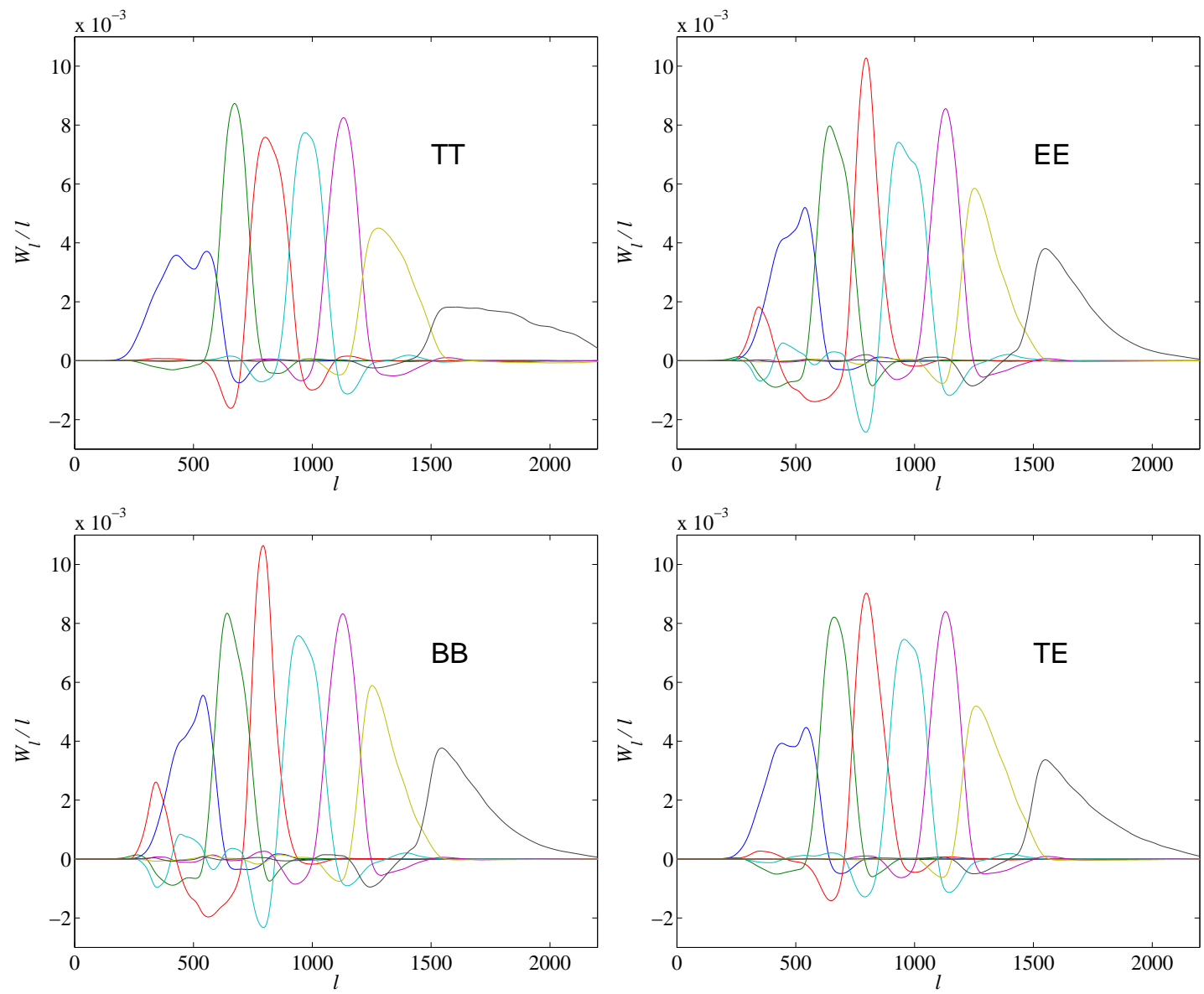

Figure 10: Band-power window functions for the CBI polarization observations with the bands shown in Table1 The expected value of band power for a given model spectrum, $C_{l}$, is $\sum_{l}[W(l) / l] l(l+1) C_{l} / 2 \pi$. The total area under each window function is equal to unity. The band powers for each spectrum also contain contributions from the the others ( $E E$ band powers are affected by changes to the model $T T$ and $T E$ as well as $E E$, for example), but for the $\mathrm{CBI}$ these cross-polarized window functions are small, with peak amplitudes of no more than a few percent of the same-polarized window functions. 

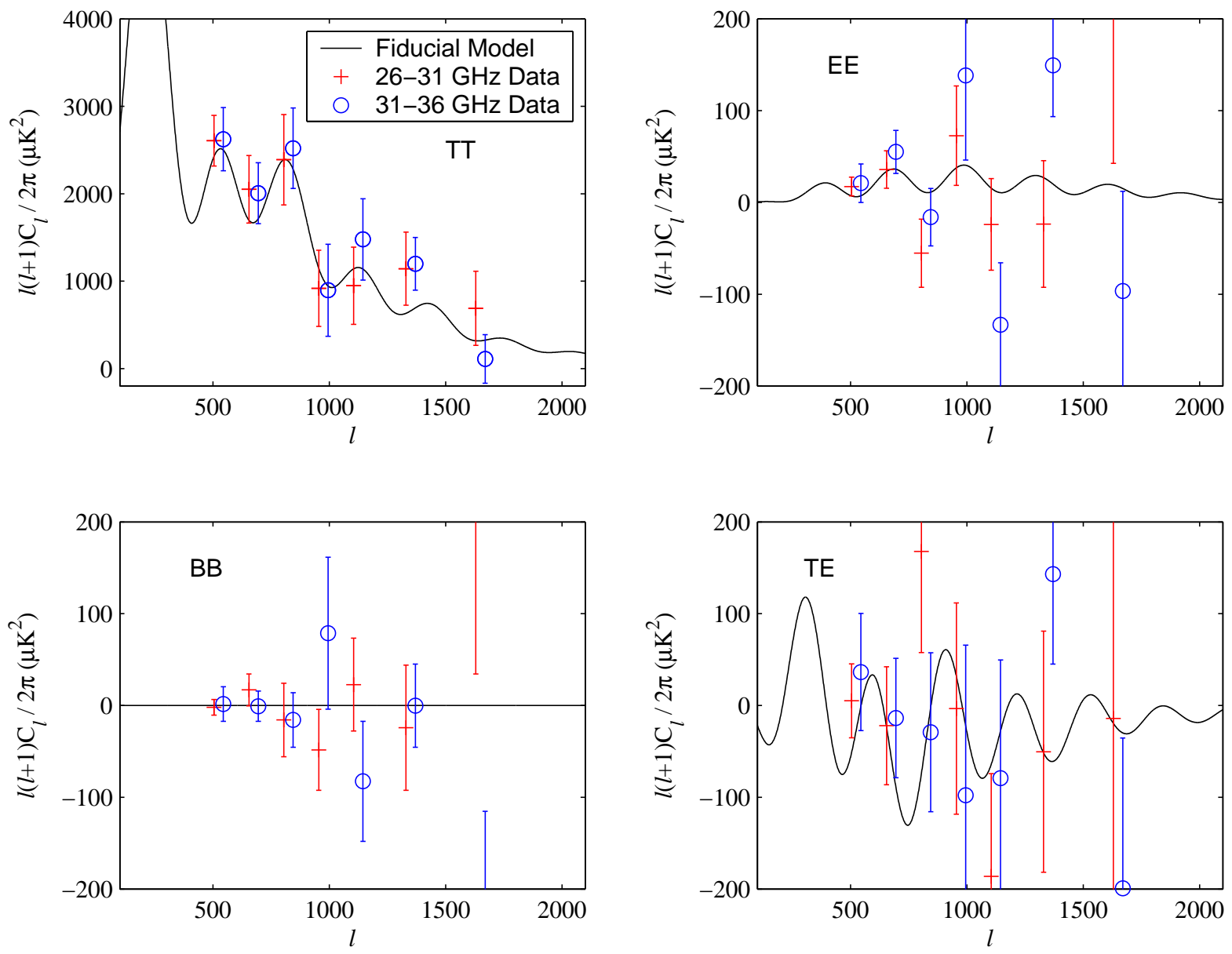

Figure 11: CBI polarization power spectra obtained from low- and high-frequency channels. Red points: 26-31 GHz; blue points: 31-36 GHz. The points have been offset horizontally in $l$ for clarity. The four panels show total intensity power spectrum $T T$, curl-free polarization mode power spectrum $E E$, curl polarization mode power spectrum $B B$, and cross-spectrum $T E$. The fiducial model curve is the same as in Fig. 6. 